Horizons philosophiques

\title{
Pluralisme linguistique et intégration européenne : les tensions identitaires de l'Union
}

\section{Irène Bellier}

Volume 12, numéro 1, automne 2001

Langue : identité plurielle

URI : https://id.erudit.org/iderudit/801195ar

DOI : https://doi.org/10.7202/801195ar

Aller au sommaire du numéro

Éditeur(s)

Collège Édouard-Montpetit

ISSN

1181-9227 (imprimé)

1920-2954 (numérique)

Découvrir la revue

Citer cet article

Bellier, I. (2001). Pluralisme linguistique et intégration européenne : les tensions identitaires de l'Union. Horizons philosophiques, 12(1), 53-86. https://doi.org/10.7202/801195ar d'utilisation que vous pouvez consulter en ligne.

https://apropos.erudit.org/fr/usagers/politique-dutilisation/ 


\section{Pluralisme linguistique et intégration européenne : les tensions identitaires de l'Union}

La dimension linguistique de l'Union européenne (UE) ne fait, au moment où l'on parle de son élargissement, l'objet d'aucun débat public bien que la nécessité de communiquer entre les Européens qui sont actuellement locuteurs de langues très diverses, soit un élément crucial de la démocratie. Parler de "réglementer la langue de l'Union» soulève, dans les milieux politiques nationaux, des discussions passionnées sur la loyauté des individus vis-à-vis de leur langue maternelle comme sur la nécessité de préserver la différence linguistique, creuset de la richesse culturelle de l'Europe. Parallèlement, les fonctionnaires européens et les acteurs de la construction économique et politique qui se confrontent régulièrement aux difficultés de la communication en plusieurs langues, développent des réflexions plus techniques sur la simplification des usages linguistiques d'une part, et la nécessité de développer l'apprentissage précoce des langues dans la perspective de la formation d'un espace de concitoyenneté d'autre part. Sans doute pour comprendre cette absence de débat populaire, nous faut-il admettre l'idée que la fabrication d'une Europe multiculturelle ne passe pas seulement par la reconnaissance de la pluralité des usages linguistiques nationaux et infranationaux. Elle suppose une transformation du rapport des individus à l'identité nationale qui a jusqu'à présent marqué le paysage linguistique européen et constitué un élément fort du cadre politique dont l'évolution est historique. Les incertitudes linguistiques du vieux continent représentent un moyen supplémentaire de penser ce qui fait de l'Union «un objet politique non identifié» (Abélès et Bellier, 1996).

Bien que l'Union ne soit pas une fédération, les enjeux de l'unification posent des problématiques assez semblables aux États qui la constituent. Nombreuses sont les études concernant les États fédéraux et les enjeux que représente l'adoption d'une langue à titre officiel. L'Inde est un cas d'école en raison de l'extrême richesse de son patrimoine linguistique et de la formule trilingue retenue par les constituants dans les années cinquante pour forger l'unité du pays. Après force débats celle-ci s'est imposée pour ne pas retenir la seule langue du colonisateur, l'anglais, comme moyen de contrer les tendances antagoniques du hindi et du tamoul dans leurs entreprises d'hégémonie régionale. Cela a permis aux pères fondateurs de la nation indienne qui compte un milliard de personnes d'appuyer 
les volontés de construction nationale sur une administration et une justice en prise avec une réalité plurielle, complexe et définitivement multilingue. Nous n'en sommes pas encore là dans l'Union des Quinze États européens qui comptent ensemble trois cent soixante quinze millions de personnes avec, dans la perspective d'un élargissement à vingt-sept États, l'incorporation de cent millions supplémentaires d'Européens, sans compter la Turquie. La question de la langue commune de l'Union n'est guère abordée dans les conférences intergouvernementales dont les résolutions en forme de traités jalonnent la construction juridique de l'Europe, ni dans l'esprit de doter l'administration de l'Europe d'un appareillage linguistique spécifique, ni sous forme d'une politique commune, assumée par l'Union et les États membres et dont les objectifs seraient lisibles par tous. Tout se passe comme si soulever la question des langues de travail et de communication devait toujours déchaîner des passions incontrôlables, comme si une adaptation linguistique au jeu européen devait ne résulter que du libre choix des individus simplement orientés par les politiques éducatives nationales.

Si l'Union s'intéresse à la question des langues européennes en tant qu'elles contribuent au patrimoine culturel commun qu'il importe de préserver - voire d'encourager pour approfondir la démocratie -, la question d'une langue de communication européenne avance masquée et suscite l'ire des polémistes les plus véhéments. À défaut d'une politique linguistique commune qui s'appuierait sur des engagements de la part des institutions communautaires comme des États pour utiliser telle langue comme moyen de communication, se profilent une série de dispositions d'ordre institutionnel, programmatique et technique, assez peu connues du public, pour caler les usages officiels et agir plus ou moins directement sur les langues en Europe. De facto, les lentes évolutions de la politique linguistique européenne sont moins le produit d'un désintérêt des gouvernants que l'aboutissement d'une stratégie pour éviter l'écueil de la référence à une langue unique, officielle.

La situation linguistique de I'Union est telle qu'il nous faut distinguer la problématique des langues officielles de celle de la langue des officiels, au regard de l'évolution du paysage linguistique européen qui est caractérisé par des tendances longues ponctuées par des soucis réglementaires réguliers. Cet article concerne d'une part la question de la langue officielle de l'Union et les problèmes que pose la pratique d'un grand nombre de langues officielles, et d'autre part des rapports entre langues officielles et identités nationales, ce qui concerne le devenir des langues nationales et le destin des langues régionales. 


\section{La question de la langue officielle et les problèmes que pose la pra- tique d'un grand nombre de langues officielles}

L'Union européenne se donne volontiers pour slogan de réaliser l' 'unité dans la diversité». Un tel slogan incite à se demander si l'unité est une perspective à définir dans le futur tandis que la diversité représenterait l'état actuel de l'UE. Quelles sont les dynamiques entre ces deux états de la société? Comment l'UE présente-t-elle cette question dans ses documents publics? De quelles façons les États membres passent-ils de l'unité linguistique nationale à la reconnaissance du plurilinguisme européen?

\section{État des lieux}

Une rapide recension des traités ${ }^{1}$ montre que la question est abordée par la voie réglementaire et que sont distingués les enjeux relatifs à la prise de décision, à la mobilisation des citoyens, à la préservation des cultures et à l'éducation. Ainsi l'article 217 du Traité de Rome (1957) dispose-t-il que "Le règlement des questions linguistiques pour les organes de la Communauté sera fait par le Conseil à l'unanimité". D'une grande brièveté, ce texte semble n'avoir pour seule fonction que de localiser la nature de la décision du côté des Etats membres. Les questions linguistiques, comme il est dit, se situent dans le domaine intergouvernemental et non dans le domaine communautaire. Elles relèvent du conseil des Ministres, non de la Commission européenne, et la règle de l'unanimité prévaut pour toute décision à prendre. Tout État peut donc opposer son veto à une proposition qui serait contraire à son intérêt national. II en résulte que sauf certaines exceptions qui montrent que ce règlement est déjà le fruit d'une négociation, la langue officielle de chaque État membre a vocation à devenir langue officielle de l'Union.

L'article 21 du Traité consolidé de l'Europe stipule que "Tout citoyen de l'Union peut écrire à toute institution ou organe visé au présent article ou à l'article 7 dans l'une des langues visées à l'article $314^{2}$ et recevoir une réponse rédigée dans la même langue.» Cet article s'inscrit dans le principe de l'égalité des langues qui est établie par le règlement no $1 \mathrm{du}$ Conseil. Si la disposition semble correcte, certains problèmes se posent quant à son application. L'article 21 pose notamment le problème de l'adéquation entre la langue dans laquelle un citoyen peut communiquer

1. http://europa.eu.int/eur-lex/fr.treaties

2. Cet article stipule que le traité dit de Rome "est rédigé en exemplaire unique, en langue allemande, en langue française, en langue italienne et en langue néerlandaise, les quatre textes faisant également foi..." et que "en vertu des traités d'adhésion, font également foi les versions du présent traité en langues anglaise, danoise, espagnole, finnoise, grecque, irlandaise, portugaise et suédoise". 
avec ceux qui ont la charge de gouverner - c'est le principe du demandeur qui prime en apparence - et la langue de la réponse qui est plus difficile à former au niveau des rédacteurs supra nationaux qui expérimentent une hybridation linguistique et culturelle. En effet, la question de la langue du citoyen ne peut être considérée sans avoir à l'esprit que les moyens donnés aux institutions auxquels il ou elle s'adresse ne suivent pas, comme on le verra plus loin en référence aux pratiques de la Cour de Justice.

Si l'on s'intéresse au règlement européen du rapport entre langue et culture qui, pour la première fois, entre dans le paysage juridique de l'Union en 1993 avec le Traité de Maastricht, on peut s'appuyer sur l'article 128, titre IX «Culture» qui précise que : «La Communauté contribue à l'épanouissement des cultures des États membres, dans le respect de leur diversité nationale et régionale, tout en mettant en évidence l'héritage culturel commun". Cet article évoque une tension entre la diversité nationale et régionale des États membres de l'Union et l'héritage culturel commun. L'énoncé, fruit d'une négociation certainement ardue, révèle que la langue est un phénomène secondaire dans l'esprit des rédacteurs de ce texte, bien que son apprentissage soit en partie soutenu par les autorités selon des choix politiques qui mettent en danger certaines langues, en en subventionnant d'autres. En effet, si l'on ne peut nier le fait que la langue contribue à l'expression de la culture, on peut se demander si chaque langue nationale fait partie de l'héritage commun. La question de la diversité des langues européennes est évidemment cruciale sous cet angle. La plupart des langues nationales du vieux continent font partie de la famille indo-européenne qui s'est constamment propagée vers l'Ouest en se mêlant, souvent violemment, à d'autres langues, elles-mêmes ciselées par l'histoire des peuples qui les portent. Sur un fond pluraliste, certaines langues dominent aujourd'hui en Europe et dans le monde, indépendamment de l'identification nationale des peuples qui les parlent. Ainsi le paysage des langues officielles en Europe se superpose-t-il à celui des langues parlées par les peuples européens qui est plus complexe encore.

Cet aspect est abordé par l'article 149 du Traité consolidé de l'UE qui, remplaçant l'article 126, stipule que : «La communauté contribue au développement d'une éducation de qualité en encourageant la coopération entre États membres et, si nécessaire, en appuyant et en complétant leur action, tout en respectant pleinement la responsabilité des États membres pour le contenu de l'enseignement et l'organisation du système éducatif ainsi que leur diversité culturelle et linguistique." L'écriture de ce texte révèle que l'on se situe au cœur du principe de subsidiarité qui 
dispose que l'action de la communauté vise à :

- développer la dimension européenne dans l'éducation, notamment par l'apprentissage et la diffusion des langues des États membres;

- favoriser la mobilité des étudiants et des enseignants, y compris en encourageant la reconnaissance académique des diplômes et des périodes d'études;

- promouvoir la coopération entre les établissements d'enseignement;

- développer l'échange d'informations et d'expériences sur les questions communes aux systèmes d'éducation des États membres;

- favoriser le développement des échanges de jeunes et d'animateurs socio-éducatifs;

- encourager le développement de l'éducation à distance3.

La rédaction montre que l'on est aux antipodes d'une politique officielle de la langue européenne. Les actions communautaires ont pour seul objet de compléter l'action des États membres et d'encourager la dimension européenne, par l'échange, la mobilité, etc. Où l'on retrouve les grandes préoccupations des politiques communautaires de mise en réseau et de liberté de circulation des hommes et des idées qui, sans être l'objet d'actions positives, bénéficient de la construction d'un cadre réglementaire. La Communauté va donc favoriser la diffusion des langues des Etats membres. Cela nous permet de souligner un aspect crucial du paysage linguistique européen qu'est la place des langues nationales dans le système de communication de l'Union.

\section{La politique des langues officielles}

La politique des langues officielles de l'Union européenne est caractérisée par une forme de compromis entre le principe de la reconnaissance des langues nationales et un principe d'économie pour essayer de réduire la diversité de l'UE, non pas au plan des références idéologiques, mais par souci d'accélérer les modes de communication entre les partenaires et les mettre en phase avec le temps de la décision qui répond à des logiques communautaires et extra-nationales.

Les choix qui ont présidé à la reconnaissance de onze langues offi-

3. Pour contribuer à la réalisation des objectifs visés au présent article, le Conseil adopte : «- statuant conformément à la procédure visée à l'article 251 et après consultation du Comité économique et social et du Comité des régions, des actions d'encouragement, à l'exclusion de toute harmonisation des dispositions législatives et réglementaires des États membres; - statuant à la majorité qualifiée sur proposition de la Commission, des recommandations." 
cielles pour Quinze pays ont été réalisés en fonction des situations linguistiques s'appliquant aux élites intellectuelles et politiques du pays, ce qui explique quelques sacrifices que l'on peut placer du côté des raisons expliquant le déficit démocratique de l'UE. On note ainsi le sacrifice sur l'autel de la Communauté du luxembourgeois (le Lëtzebuergesch), qui a un statut de langue, au motif que l'administration et la classe politique luxembourgeoises travaillent en français et en anglais. Ce pays a donc concédé l'usage du français sur la scène européenne. Du côté de la Belgique, un mouvement semblable a été enregistré en raison de la proximité entre le flamand et le néerlandais, le wallon et le français. Du côté irlandais des rapports historiques bien que tendus ont abouti à l'usage de l'anglais sur la scène européenne, avec l'exigence toutefois que les traités soient traduits en gaélique / irlandais. Enfin l'autrichien n'a pas été retenu comme langue officielle en raison de sa proximité avec l'allemand.

L'Union reconnaît donc douze langues pour ses traités qui sont paraphés par les Quinze représentants des États membres. La forme des signatures est très instructive car on y voit que très officiellement, la scène linguistique ne recoupe qu'imparfaitement le découpage en scènes nationales. II y a donc accord pour décoller l'univers de la langue de la représentation de la nation, ce qui montre une évolution. Mais on est loin de simplifier les usages comme le réclament beaucoup d'Européens, au vu notamment de la manière dont le problème se complique avec l'entrée des prochains États. Au registre des signatures, on observe que les pays sont rangés dans l'ordre alphabétique de leur nom dans leur langue : Belgique, Danmark, Deutschland, Ellas, España, France, Ireland, Italia, Luxembourg, Nederland, Osterreich, Portugal, Suomi, Sverige, United Kingdom. On voit simultanément que des nuances supplémentaires sont indiquées pour la Belgique afin que la signature du plénipotentiaire engage les communautés française, flamande, germanophone, la région wallonne, la région flamande et la région de Bruxelles capitale - ce qui se dit en trois langues, français, néerlandais et allemand, avec deux déclarations encadrant le paraphe : "pour sa Majesté le roi des Belges" et "Cette signature engage la Communauté, etc.". De même pour l'Irlande, la motion "For the President of Ireland" est traduite en irlandais. Ces petites notations montrent que sur les plans protocolaire, diplomatique et juridique des efforts sont produits pour engager les communautés nationales dans l'acte international contraignant pour la signature duquel elles ont délégué l'un des leurs, choisi par le système national de représentation. Cet engagement se formule dans la langue qui est devenue, dans l'histoire des États, le symbole de l'identité et de la loyauté : la langue 
standard consacrée officielle.

On voit donc que les Traités sont la partie visible d'un rapport de force qui s'est joué antérieurement, sur d'autres scènes : d'une part, la scène de la négociation à laquelle les citoyens ne sont pas conviés, d'autre part la scène politique nationale à laquelle ils participent par la voie de leurs représentants politiques et qui conduit à ce que des efforts spéciaux soient produits pour que des entités culturelles et linguistiques telles que les communautés wallonne et flamande, la communauté germanophone, distinguées des entités politiques telles la région, wallonne, flamande ou Bruxelloise, se sentent non seulement concernées mais engagées par la signature du Roi des Belges.

On constate ainsi que la langue officielle ne reflète qu'imparfaitement la situation linguistique de chacun des États et celle de l'Union Européenne en particulier. La scène européenne révèle l'état des rapports de force politiques qui se jouent au sein des États pour transformer la donne héritée de l'histoire des États nations.

\section{La diversité linguistique vue du côté des institutions européennes}

La diversité européenne est un acte de foi ${ }^{4}$. L'Union européenne ne serait pas ce qu'elle est, si elle n'avait refusé pour le vieux continent le principe de domination d'un État sur les autres. L'Union n'étant ni un empire ni un État nation - deux modèles par lesquels l'Europe a construit son identité intérieure et extérieure, y compris linguistique - il lui faut mettre en avant le principe de la diversité de ses membres, moyen qui lui permet de ne pas trancher la question de la forme nationale ou fédérale de l'entité qui se construit.

Le premier symbole de cette diversité est celle des langues pour une raison sur laquelle s'accordent la plupart des linguistes, à savoir que la langue est "un badge identitaire». (Sue Wright, 1999). Badge et donc symbole d'identité, la diversité linguistique s'exprime dans l'espace public, c'est-à-dire dans l'espace des rencontres, et beaucoup plus rarement dans l'espace privé, domestique ou intime. Dans l'Union européenne, elle

4. La diversité de l'UE comprend plusieurs dimensions qui permettent d'expliquer les soubresauts de l'unification et d'appeler à un plus grand effort dans l'éducation plurilingue. Mais la mise en place de l'Europe ne se limite pas pour les citoyens européens à l'apprentissage d'une langue étrangère; il leur est plus important de savoir se repérer dans le paysage de la communication multilingue qui aboutit, comme on le voit régulièrement avec les textes de la Commission, à des propositions peu intelligibles dans les registres d'interprétation nationaux (Bellier 2001). Par ailleurs, l'argument consistant à invoquer la diversité linguistique comme facteur explicatif des difficultés à prendre une décision - ou de l'inanité de celle-ci - permet de ne pas évoquer sur la place publique des facteurs plus occultes telles que les pressions nationales ou celles des lobbies. 
concerne au premier chef les langues de rédaction des traités qui sont au nombre de douze : allemand, anglais, danois, espagnol, finnois, français, grec, irlandais, italien, néerlandais, portugais et suédois. L'irlandais a le statut particulier de langue officielle de traité, en raison de sa position de première langue officielle en Irlande bien qu'elle soit parlée par une minorité de personnes et que certains la considèrent comme en voie de disparition, en raison de la prévalence de l'anglais, admis comme seconde langue officielle.

Ce type de reconnaissance qui introduit une subtile distinction dans la notion de langue officielle a été demandée par le Parlement européen pour le catalan dont les représentants, qui ont gagné la bataille de la langue et de l'éducation dans leur région en Espagne, sont extrêmement actifs au niveau européen. Leur principal argument est que sept millions de personnes parlent le catalan, ce qui en fait une minorité linguistique conséquente par comparaison avec d'autres peuples dont la langue a acquis le statut de langue officielle comme c'est le cas pour les petits États membres : citons par exemple le danois ou le finnois avec cinq millions de locuteurs environ. La reconnaissance d'une langue au niveau européen est le premier pas vers l'officialisation de la langue à l'extérieur de la région qui la parle, la Communauté autonome de Catalogne dans le cas cité.

Le principe de la reconnaissance officielle de la diversité des langues constitutives de l'Europe, ainsi que l'observation de ce qui se passe sur le terrain, montre qu'il n'y a pas de langue dominant le linguascape européen pour paraphraser Arjun Appadurai (1996). La situation actuelle est caractérisée par différents types de situations évoluant à des rythmes différents.

Le premier type de situation est illustré par les cinq institutions européennes qui fonctionnent sur la base d'un plurilinguisme régi par différents principes selon leur fonctionnalité et pour lesquelles il nous faut distinguer entre langues officielles et langues de travail. Trois institutions incarnant l'une la souveraineté du peuple (Parlement européen), l'autre la légitimité des États (Conseil des Ministres), la dernière, la Justice, vivent un double multilinguisme. Le premier fonctionne au niveau le plus officiel sur le registre des onze langues officielles des États. Le second résulte de l'usage de quelques langues pivots dans différentes circonstances laborieuses, lorsque les intervenants ne peuvent avoir recours aux services complets d'interprétation. Ce système est propice à l'expression des parlementaires qui, en qualité de représentants du peuple, ne tirent pas leur légitimité de leur position au centre de l'UE. Leur mission étant 
d'exprimer l'une des voix de la diversité, il est admis qu'ils ne soient pas nécessairement compétents dans les langues principales de l'UE pour exercer leur fonction. Le conseil des Ministres connaît une situation semblable, les ministres ayant pour mission de représenter les États dans leur secteur de compétence, si bien que le principe de l'égalité a été retenu au niveau linguistique. Pour la Cour de Justice, le principe du multilinguisme le plus large est retenu - en raison de l'article précité par lequel tout citoyen a vocation à saisir l'Union dans la langue de son choix. La Cour a pour devoir de suivre la procédure dans la langue par laquelle le cas est posé aux magistrats, mais elle prévoit des mécanismes intermédiaires de travail en français. Cela est dû au rôle historique de la France dans la construction juridique de l'Union et à la place du français dans la production du droit continental. Dans ces trois instances se posent d'innombrables problèmes de traduction qui induisent régulièrement des situations dans lesquelles les interlocuteurs s'expriment en deux ou trois langues seulement et des propositions pour travailler sur un registre simplifié. Pour les deux autres institutions européennes, la Commission et la Cour des Comptes, qui ne sont pas chargées de fonctions de représentation de la diversité européenne - mais qui incarnent la tendance contraire de l'intégration européenne en étant l'une -, la source de l'initiative européenne et la garante des traités, l'autre chargée de l'audit des comptes et de l'apurement du budget, le principe de la simplification domine d'emblée les procédures. Trois langues de travail ont été reconnues, le français, l'anglais et l'allemand, pour simplifier les échanges et la communication inter-linguistique sans imposer un monolinguisme, "politiquement incorrect» dès la fondation de la Communauté par le Traité de Rome.

Le second type de situation est représenté par la rencontre des dispositions officielles et des usages linguistiques qui suscite une multiplicité de configurations particulières, dont on peut se faire une idée sur le terrain des institutions précitées où l'on observe l'émergence d'un langage commun que les spécialistes appelleraient sans doute un sociolecte et qui est très instable (Bellier $1999 \mathrm{a}, 2001$ : 27-28). Le lecteur peut avoir un aperçu de cette instabilité en consultant le site Internet de la Commission qui met «l'Europe en ligne», titre du portail d'accès du serveur Europa en usage depuis mars 2000. Cette expression déclinée dans les onze langues officielles remplace le message de bienvenue autrefois formulé dans les différentes langues (Wilcommen, Welcome, Bienvenue, Bienvenido, Benvenido, Tervetuloa, etc.) Le service gestionnaire de la nouvelle stratégie de communication de la Commission qui change régulièrement ses mises en pages et son mode de navigation, introduit des 
documents dans les différentes langues officielles sans y parvenir uniformément dans tous les domaines. On observe donc des grands trous et des passerelles, ce qui de facto conforte certaines langues dans une position centrale. Selon les Directions générales et les dossiers, l'internaute peut avoir accès à un texte référencé dans les onze langues ou dans l'une d'elles seulement, alternativement anglais et français. C'est le cas par exemple des discours des commissaires ou des organigrammes, ce qui permet de mesurer la marginalisation de l'allemand, bien que cette langue parlée par plus de quatre-vingt-huit millions de personnes, soit le plus grand groupe de locuteurs natifs, ait été reconnue langue officielle de l'Allemagne et de l'Autriche et langue de travail de la Commission. Le fait multilinguistique aboutit, dans les services de la Commission qui est l'instance la plus intégrée sur le plan culturel (Abelès et Bellier 1996) à des constructions orales et écrites tout à fait fantaisistes, liées d'une part à l'inégale compétence des agents dans chacune des trois langues de travail reconnues, d'autre part à la nature même du travail qui stimule la production d'un jargon professionnel mêlant, par souci d'économie, des termes de différentes langues mieux appropriés que la traduction à la réalité que la Commission souhaite faire advenir.

Le troisième type de situations se présente au-delà de la scène institutionnelle de l'Union, sur le terrain des individus, dans le quotidien des locuteurs - auditeurs, là où règne la diversité qui forme le terreau des constructions linguistiques nationales. Celles-ci évoluent indépendamment des institutions, mais elles peuvent être stimulées par elles. Ainsi, par exemple, enregistrait-on en 1995, un début d'intérêt pour favoriser l'apprentissage des langues européennes dans le cadre de la construction de "la société cognitive», que la Commission s'efforce de mettre en place dans Le Livre Blanc qu'elle publie à cette date. Le quatrième objectif de ce livre d'intention est de "maîtriser trois langues communautaires". Cela est présenté comme la "condition indispensable pour permettre aux citoyens de l'Union de bénéficier des possibilités professionnelles et personnelles que leur ouvre la réalisation du grand marché intérieur sans frontière.» On constate qu'avant d'être objet et instrument de connaissan$c e$ la capacité linguistique a pour fonction de stimuler une faculté d'adaptation à des milieux de travail et de vie marqués par des cultures différentes. Dans l'esprit européen qui prévaut à la Commission, la langue est un point de passage nécessaire pour la connaissance des autres et sa maîtrise contribuera "à renforcer le sentiment d'appartenance à l'Europe dans sa richesse et sa diversité culturelle, et la compréhension entre les citoyens européens. Le plurilinguisme est un élément constitutif tant de 
l'identité et de la citoyenneté européennes que de la société cognitive." Cette prise de position se complète par une évaluation très positive de l'action de l'Union dans le développement de l'apprentissage des langues via le programme LINGUA, qui a été intégré dans les programmes SOCRATES et LEONARDO. On notera que les acronymes de ces programmes culturels sont intelligibles dans la quasi totalité des langues européennes et rendent hommage au patrimoine commun que sont les grands noms attachés à la civilisation européenne. L'idée de la Commission est de populariser l'apprentissage des langues pour qu'il ne soit plus l'apanage des élites ou des voyageurs et de développer l'enseignement des langues dans le primaire et le secondaire en prenant modèle sur les Écoles européennes. Elle encourage donc l'Union à soutenir les programmes nationaux allant dans ce sens, comme ce fut le cas des classes européennes inégalement développées dans le paysage scolaire français, au motif qu'elles étaient un moyen détourné de sélection des meilleurs. Cinq ans après, l'apprentissage des langues communautaires est devenu une priorité dans l'agenda du ministre français de l'éducation nationale.

\section{Le multilinguisme européen}

Un sondage Eurobaromètre 5 réalisé en 2000 fait état d'une nette progression des Européens ${ }^{6}$ vers le multilinguisme, avec une préférence pour l'anglais comme seconde langue par rapport au français et à l'allemand. Il estime que $45 \%$ des Européens sont capables de prendre part à une conversation dans une langue autre que maternelle, avec de grandes disparités entre le Luxembourg où cela concerne la quasi totalité de la population, les Pays Bas, le Danemark et la Suède où la population est plurilingue à $80 \%$, le taux chutant à moins de $30 \%$ pour le Royaume Uni, l'Irlande et le Portugal. L'anglais est parlé par $47 \%$ des citoyens européens, suivi par l'allemand $32 \%$, le français $28 \%$ et litalien $18 \%$, au titre de première ou de deuxième langue. Le français, pour $37 \%$ des citoyens et l'anglais pour $69 \%$, sont considérés comme les deux langues les plus utiles à connaître. La proportion des habitants capables de s'exprimer en langue étrangère va de $77 \%$ des étudiants, décroissant chez les cadres et les jeunes jusqu'à atteindre $19 \%$ des moins de 15 ans, avec une dispersion socio-démographique assez homogène : $47 \%$ des hommes, $43 \%$ des femmes, $45 \%$ de la moyenne des Etats membres, $40 \%$ des chômeurs, 31 \% des personnes au foyer, etc.

Les politiques nationales d'éducation linguistique semblent converger

5. http://europa.eu.int/comm/education/languages/tr/lang/europeanlanguages.html

6. L'organisme sondeur qualifie les sujets de son étude de "citoyens" ou "habitants". 
vers une formule tri-linguistique qu'Eurobaromètre fait ressortir pour chacun des pays, ce qui révèle la diversité des usages. Les choix des deuxième et troisième langues tiennent à l'histoire du pays comme à sa localisation, ce qui fait que la langue des voisins est en général choisie. L'encouragement à l'éducation plurilingue à un âge précoce peut être lié aux recommandations programmatiques de la Commission qui s'appuie sur un début de réflexion sur la langue comme moyen d'expression, comme mode de communication et comme fondement de la citoyenneté européenne. Toutefois les évolutions qui se dessinent dans le paysage européen s'observent alors que les textes européens ne sont pas axés sur une formule linguistique propre à l'Union qui établirait un centre, marqué territorialement et linguistiquement. II s'agit plutôt d'adapter les individus à la communication dans un espace plus large que le cadre national et à la possibilité de se référer à plusieurs centres.

On observe ce point avec la Charte des Droits fondamentaux de l'Union adoptée en décembre 2000 à Nice, dont l'article 22 stipule que "L'Union respecte la diversité culturelle, religieuse et linguistique." C'est la seule chose dite sur la question de la langue tandis que les différents articles évoquent toutes sortes de droits, l'article 40 précisant que «Tout citoyen et toute citoyenne de l'Union a le droit de vote et d'éligibilité aux élections municipales dans l'État membre où il ou elle réside». Cela suppose une certaine compétence linguistique dans la langue de l'État de résidence, qui n'est pas explicitée dans ce texte, à charge pour les États de fixer la règle de la résidence et pour les individus de s'y plier.

Au début des années 90 , la Communauté économique européenne admettait neuf langues officielles dont l'interprétation et la traduction supposaient de prévoir des mécanismes de travail à partir de soixantedouze paires linguistiques, ainsi que l'on dénomme le passage du français vers l'anglais, du danois vers le portugais, du grec vers l'espagnol, du finnois vers l'allemand, etc. Depuis 1995, l'intégration de trois nouveaux États membres dans l'Union a élargi le nombre des langues officielles à onze, augmentant de ce fait le nombre de paires linguistiques à cent dix. Si l'on comptait tous les candidats possibles à l'intégration européenne, y compris ceux dont la candidature n'a pas encore déposée (Chypre, Islande, Liechtenstein, Malte, Norvège, Suisse, Turquie, Albanie, Biélorussie, Bulgarie, Croatie, Slovaquie, Slovénie, Estonie, Hongrie, Lettonie, Lituanie, Pologne, République Tchèque, Roumanie, Russie et Ukraine), le nombre de combinaisons possibles s'élèverait à huit cent soixante-dix (Born 1996). Un tel nombre signifierait que chaque État membre devrait, pour pouvoir être opérationnel dans le 
cadre de l'Union, envoyer des quantités de linguistes, ce qui semble impossible pour les plus petits d'entre eux, comme pour Malte, par exemple qui ne compte que 300000 personnes. Plus réalistes sont les perspectives à moyen terme, c'est-à-dire à partir de 2004, d'un élargissement plus limité de l'Union qui entraînerait l'inclusion d'au moins cinq langues officielles. De ce fait, peut être déjà prévue l'augmentation du nombre de paires linguistiques de cent dix à deux cent quarante. Bien que des signaux soient régulièrement adressés par les équipes de traducteurs, il semble que les institutions européennes aient du mal à évaluer cet aspect de l'élargissement.

Face au risque, annoncé par la Commission Prodi durant l'été 2001, de ne recourir qu'à l'anglais comme langue de communication européenne, le Parlement européen vient cependant de voter une résolution refusant le principe de l'élimination de certaines langues tout en acceptant le principe du relais de la traduction par quelques langues pivots 7 . II s'agit là d'une formule de compromis pour satisfaire les États membres et les États candidats qui ne souhaitent pas voir leur langue traitées comme des langues de seconde catégorie, et pour simplifier les procédures sans recommander l'usage unique de l'anglais, comme le propose la Commission. Du côté des États membres, le problème linguistique n'est pas une priorité de la réflexion sur la redéfinition du projet européen qu'impose le fonctionnement d'une grande Europe. Du côté des États candidats, le problème n'est pas directement abordé dans le cadre des négociations qui se limitent à la reprise de l'acquis communautaire et à l'adaptation de leur capacité administrative pour faire face aux défis de l'appartenance à l'Union. Mais, en l'état actuel de reconstruction des identités nationales à laquelle procèdent les pays candidats, notamment ceux qui sont sortis de l'orbite soviétique, il n'est pas question de renoncer à ce symbole de l'unité retrouvée qu'est la langue nationale. La demande de reconnaissance de ces langues est donc légitimement posée devant les institutions européennes. Par conséquent restent également posés les problèmes de la qualité de la production écrite et orale comme celui du coût induit par la traduction et interprétation en seize langues officielles.

En ce qui concerne la qualité, l'expérience montre que la simplification des usages linguistiques que représente l'interprétation par la voie d'une langue relais, induit une série de décalages propices au développement des malentendus chez les auditeurs d'une enceinte multilingue telle que le Parlement Européen. C'est la raison pour laquelle il est d'usage de

7. Libération, Le Monde 5 septembre 2001. 
dire que «les Danois sont toujours les derniers à rire», leur langue ayant été stigmatisée comme la plus éloignée des autres dans l'ensemble des onze langues officielles (Abélès, 1992). D'autres mesures qui ont été adoptées par les agents de la Commission pour pallier l'insuffisance des ressources humaines du service de traduction, aboutissant ici à des traductions sur une base totalement ad hoc, là à des improvisations génératrices de contresens, sont responsables du caractère très indigeste des textes institutionnels dont les versions initiales sont rarement intelligibles de prime abord. Enfin, les tentatives visant à recourir à la traduction automatique pour accélérer et désengorger le traitement des dossiers européens se sont soldées par tant de faux amis que les exemples pourraient devenir une source inépuisable de plaisanterie. Un journaliste au Monde a ainsi relevé le danger que représentaient "des avions sans pilote qui prennent pour cible les centrales nucléaires à basse altitude», alors que le texte original parlait des «avions qui survolent les centrales en pilotage automatique" (La Guérivière 1992 : 51).

Si l'on comprend que la communication écrite et orale dans un cadre multilinguistique est une affaire complexe, on s'explique mal pourquoi les institutions qui sont attachées à la production d'actes normatifs ne prennent pas toute la mesure des enjeux linguistiques qui se nouent autour de cette production et de l'interprétation nécessaire à la construction d'un espace politique, juridique et communicationnel européen.

Si les coûts d'une traduction élargie dans le cas de 21 langues officielles ont été chiffrés par le Parlement Européen à 520 millions d'Euros par an, le problème ne relève pas du simple traitement budgétaire de la question, bien que cet aspect soit crucial pour procéder au recrutement d'un plus grand nombre de linguistes (traducteurs - interprètes) pour faire face aux besoins. Sans doute faut-il aussi réfléchir de manière plus approfondie au fonctionnement de la langue, à ce que l'on attend de la langue dans la production de l'Europe. Le cas du service de traduction de la Cour Européenne de Justice a fait l'objet d'une analyse particulière, au regard des problèmes auquel il a été confronté dans les élargissements successifs de l'Union et de ceux qu'il anticipe dans la perspective d'une Europe élargie. Cette étude de cas peut nous servir d'exemple pour les autres institutions européennes.

À la Cour de Justice, où règne la règle de l'égalité des langues (Règlement no 1 du Conseil des Ministres), certains documents doivent êtres traduits dans toutes les langues officielles (actuellement au nombre de douze, avec l'irlandais qui n'a jamais été utilisé) et le français a été adopté comme langue de travail interne. Si l'égalité des États membres 
sur la scène juridique est essentielle à la légitimité de la Cour, la charge de travail actuelle porte sur la traduction de 325000 pages de documents légaux complexes par an. En raison des obligations de traduction, l'instruction d'un cas nécessite dix-huit à trente-six mois et des délais supérieurs à deux ans sont enregistrés pour publier les décisions.

Selon Mullen (2000), la solution de cette question dépend du Conseil et de la Commission pour régler sur le plan politique la question du nombre des langues officielles admises à la Cour, et du Parlement Européen pour voter le budget nécessaire à l'augmentation des moyens du service de traduction. La Cour de Justice ne dispose d'aucun autre moyen autonome que l'accroissement du rendement des traducteurs pour répondre à la demande de justice. De fait, les différents élargissements ont été "digérés» par le service de traduction sans qu'il ait été ni réorganisé ni considérablement augmenté de taille. Actuellement, chaque langue officielle est traitée par une division composée d'un chef, d'une vingtaine de juristes - linguistes, aidés par 6 à 10 secrétaires et correcteurs. Tous les linguistes traduisent vers leur langue maternelle, directement.

La situation de la Cour est donc différente de celle du Parlement européen qui a adopté un système de "hub" pour l'interprétation, trois langues principales (anglais, français et allemand) étant utilisées comme noyau central-relais. Ce système présente l'avantage de diminuer le nombre de combinaisons linguistiques possibles et linconvénient de faire disparaître les nuances de la langue. II ne peut donc être appliqué à la Cour de Justice, les nuances et l'exactitude des traductions étant essentielles au niveau légal. La langue dans laquelle le cas est posé est utilisée pour plaider et pour communiquer les observations relatives au cas. Pour les procédures en action directe, la partie demanderesse choisit la langue du cas mais la langue du défendeur peut être aussi utilisée. Comme les juges et les avocats travaillent en français, le service de traduction se concentre sur les documents en rapport avec la substance du cas. Le suivi du cas entre la Cour et les États membres induit donc différentes étapes de traduction dans les différentes langues officielles et dans la langue de travail interne.

Avec l'élargissement, ce service a fait l'expérience d'une manière expéditive d'adapter le service de traduction aux besoins du finnois et du suédois. En l'absence d'une préparation adéquate, le service a dû procéder en dix jours à la traduction des décisions finales de la Cour. Si l'on peut compter sur une "adaptabilité" accrue des personnels de traduction pour reprendre le langage du management, il semble que le problème de la traduction des textes à portée légale se pose bien avant 
l'entrée dans le club des États membres. Tout commence à se jouer durant la phase de reprise de l'acquis communautaire, lorsque les États candidats doivent traduire - pour pouvoir les transposer dans leur système légal national -, la totalité des textes réglementaires et légaux de l'UE.

Dans le cas de la Suède et de la Finlande - sans parler de l'Autriche qui s'appuie sur l'allemand - les problèmes ont été assez vite résolus, ces deux pays ayant déjà l'expérience du système de marché et des structures administratives et légales solides. II n'y avait pas d'obstacles majeurs sur le plan de l'interprétation. Mais cela risque de ne pas être le cas des Pays d'Europe Centrale et Orientale qui doivent ingérer un acquis communautaire très juridique en évacuant simultanément les systèmes antérieurs d'interprétation et en réformant les pratiques des juristes. Dans le langage de la Commission, cela pose le problème crucial de l'adaptation de leurs "capacités administratives" à la mise en œuvre des obligations de l'intégration. Dans cette vaste entreprise de réorganisation des fondamentaux sur lesquels repose l'État de droit à l'européenne, les représentants des États candidats qui sont souvent pris au dépourvu par les exigences de la Commission, s'appuient sur des bureaux d'étude, parfois juridiques parfois tout autre, pour comprendre, traduire, interpréter, réformer. L'expérience prouve que la traduction des textes peut avoir été réalisée, dans le pays même, par des linguistes peu compétents en loi communautaire ou même en droit international. Or leur traduction sera validée comme officielle lors de l'adhésion. Le problème s'est posé pour la Finlande mais pas pour la Suède et il risque de se poser avec n'importe lequel des pays candidats.

Comparé à ces questions d'interprétation, le passage à deux cent quarante combinaisons linguistiques pose un plus grand nombre de problème encore, sur le plan de l'organisation et pour mettre sur pied des équipes efficaces. Si le service restait en l'état, vingt linguistes seraient responsables de seize langues alors que le nombre de combinaisons possibles dépasse la capacité humaine. Laissant à part ce qui correspondrait aux questions budgétaires et aux perspectives organisationnelles, il est devenu prioritaire de se concentrer sur la réforme des usages de la langue.

Dans l'impossibilité de revenir sur le principe de l'égalité des langues qui fonde la légitimité de la Cour, différents moyens commencent à être envisagés. Le premier serait de développer le système de hub pour réduire le nombre de combinaisons possibles, bien que cela risque d'atténuer l'exactitude du texte et d'en déformer la portée. Le second moyen vise à 
réduire le nombre de documents en circulation, en simplifiant les procédures. Enfin, certains spécialistes du droit commencent à réfléchir à un système décentralisé, reposant sur plusieurs niveaux de circuits d'instruction et de décision, à la manière américaine. La justice s'appuierait dans ce contexte sur une structure fédérale à plusieurs instances, la Cour Suprême étant chargée d'arbitrer les conflits entre les circuits. Cela reviendrait à construire dans les États membres des organes judiciaires de statut communautaire ou bien national, pour traiter des éléments préliminaires dans leur propre langue. Le niveau supérieur, celui de la Cour Européenne de Justice, ne serait saisi que lorsqu'il y aurait conflit d'interprétation.

Une telle réforme des structures judiciaires qui pourrait s'étendre aux structures politiques s'inscrit dans une perspective fédérale qui est loin de faire consensus entre les États membres. Elle devrait faire partie de la définition d'un projet pour la Nouvelle Europe qui serait discuté d'ici à 2004 autour d'un projet de constitution européenne qui introduira quelques lignes au chapitre des langues officielles. Mais pour l'instant, rien n'est dit sur le sujet. Or on voit bien que la réforme des institutions de l'Union à laquelle songent depuis Maastricht les gouvernements des États membres, ne pourra se réaliser sans réforme linguistique.

Langues officielles, identités nationales et le destin des langues minoritaires

Le problème linguistique de l'Europe qui se construit comme une entité politique (Union européenne) dérive de la consolidation historique des ensembles politiques en États nations, de type unitaire. Chacun de ces États considère sa langue officielle comme devant être légitimement reconnue et usitée dans le cadre européen. Cependant des évolutions doivent être prises en compte, certaines langues étant plus parlées que d'autres. En compétition sur la scène européenne, deux langues sont utilisées quotidiennement, le français et l'anglais, bien que le français ait commençé de perdre sa prééminence au moment où il fallut traduire les directives dans toutes les langues des États membres. Le principe de l'égalité des États sur le plan linguistique vient en effet directement contester le principe du recours à une langue centrale dans un système de communication élargi. Le français reste privilégié par la localisation des institutions en territoire francophone, mais il est menacé par l'expansion de l'anglais liée aux élargissements successifs. Mais en aucune manière ces deux langues ne sont langues de communication pour l'ensemble des citoyens européens. Devenu premier moyen de communication en Europe, l'anglais n'est pas le premier langage parlé, cette place 
revenant à l'allemand parlé par plus de quatre vingt millions de personnes. L'anglais est le premier second langage, une position qui résout la rivalité du français et de l'allemand pour cette seconde place et confirme la centralité de cette langue dans le monde. II est intéressant de suivre le concept de "centralité", développé par Abram de Swaan (1995), dans ses rapports avec les langues officielles pour comprendre que ce qui se joue sur la scène européenne est en quelque sorte préfiguré par la manière dont la diversité linguistique a été réprimée dans le processus de fabrication de l'état nation de type unitaire. Cependant l'Union ne fonctionnant pas comme un Etat unitaire, de grandes différences peuvent être observées qui ont des conséquences sur le plan linguistique.

Le besoin qu'eurent les États de communiquer et de travailler dans un langage connu de tous, ce qui aboutit aux entreprises d'unification linguistique et de formation de la langue officielle au niveau national et central, se fait aujourd'hui sentir au niveau européen. L'Union poursuit une politique de mise en réseau, dans le domaine des transports comme dans celui des nouvelles technologies de l'information et de la communication; les représentants des États membres et des institutions européennes sont à la recherche permanente des termes de compromis pour l'instruction et la mise en œurre des directives communes; les besoins de standardisation et d'harmonisation sont au cœur des entreprises proprement européennes comme on le voit à propos des négociations concernant la reprise de l'acquis communautaire par les pays candidats préalablement à leur adhésion. Le besoin d'une langue unique se fait sentir dans une série de situations que le terme de Babel vient souvent qualifier 8 .

Simultanément l'élargissement des cadres de référence des citoyens (lié à l'éducation massive, au développement des médias, à la mondialisation) ainsi que l'approfondissement de la démocratie induit par la politique de revitalisation des espaces infra-nationaux (les pays, les régions, les terroirs) qui s'appuie sur diverses formes de reconnaissance des pouvoirs locaux, y compris au niveau linguistique, relancent une dynamique dans le sens du pluralisme. De ce fait, le débat linguistique européen se pose moins en terme de centralité et de périphéries qu'en termes de capacité des individus à passer du monolinguisme au plurilinguisme.

Centralité d'une langue, officialité et diversité linguistique

Pour Abraam De Swaan (1995), socio-linguiste hollandais, le langage ne résulte pas du politique seulement. II est le produit des préférences individuelles pour apprendre une autre langue ou le résultat inattendu des

8. «Bruxelles, Nouvelle tour de Babel : Sprachen Usted the pidgin european? Non mercil» Courrier International $n^{\circ} 547: 49$. Article du Frankfuter Allgemeine Zeitung. 
décisions institutionnelles pour retenir une langue dans l'enseignement scolaire ou en écarter une autre. Pour lui, en l'absence de politique commune, le langage des institutions européennes évolue à l'aveugle. II ancre son analyse dans le paysage linguistique mondial où il compte quatre milles langues parmi lesquelles il distingue la constellation des langues du monde, une configuration qui procède de l'apprentissage d'une langue étrangère. Certaines langues sont plus demandées que d'autres et parlées par plus d'un million de personnes. Au nombre de cent quarante dans le monde, elles incluent $90 \%$ de la population. Parmi celles-ci plusieurs langues sont demandées parce qu'elles sont choisies comme seconde langue par beaucoup. La communication passe alors par le relais d'un tiers langage qui devient central. C'est cette langue pivot qu'il est important d'identifier en Europe.

Certaines langues moins parlées que d'autres restent centrales. C'était le cas du latin médiéval, parlé par un tout petit nombre de locuteurs, dont la centralité provenait du fait que la majorité de ceux qui étaient compétents dans plus d'une langue, parlaient latin. Une langue centrale dans un sous-système de communication peut être périphérique dans un autre sous-système : tel est le cas du néerlandais à l'extérieur des Pays-Bas. Ce pourrait être le cas du français dans un futur proche, notamment s'il n'était plus reconnu comme langue des institutions transnationales, mais pas celui de l'anglais qui devient central dans l'UE et pour le reste du monde.

Ces faits amènent à proposer pour comprendre le système linguistique européen, le modèle d'une galaxie dont les planètes entourées de lunes dessinent différents types de centralité. Les mouvements qui agitent la constellation sont autant le produit des développements démographiques qui permettent l'extension des langues maternelles à un plus grand nombre de locuteurs et sur de plus vastes espaces, que des décisions politiques pour enseigner telle ou telle langue, décisions qui affectent le rôle des élites dans le système politique. En effet, lorsque les élites ont le monopole de l'apprentissage des, et de l'éducation aux, langues du pouvoir, elles contrôlent les possibilités des masses à devenir autonomes, et leur propre fonction dans la société politique. On note que sur la scène européenne, les élites nationales et régionales, peu à peu impliquées dans les mécanismes décisionnels européens, sont les premières à s'européaniser, tant pour ce qui désigne leur univers de connaissances, la structuration de leurs réseaux (Bellier 1999 b) et leur capacité à maîtriser plusieurs langues dont l'anglais en commun, que pour l'extension de leurs territoires d'action. Leurs choix semblent non 
seulement voisins de ce qui se pratique dans les cercles proches de la prise de décision européenne, mais également influencer les institutions de Bruxelles. L'anglais est devenu leur seconde langue commune.

En ne citant que certains traits majeurs des grandes évolutions européennes, on observerait qu'il n'y a pas de place pour plusieurs langues centrales et dominantes dans le fonctionnement européen. Les perspectives d'unification se sont toutes accompagnées d'une réduction de la diversité linguistique à mesure que se structuraient les États modernes. Le latin fut la langue centrale de communication dans l'espace du christianisme, dont le continent européen, jusqu'au moment où la réforme poussa à la traduction de la Bible en langue vernaculaire, stimulant le lettrisme dans ces langues, ce qui fut facilité par l'invention technique de l'imprimerie (cf. Anderson 1983). Ce mouvement conduira à la fondation des académies de défense des langues en France (1635), en Angleterre (Royal Society for Improving the English Language, 1664), en Italie (Accademia della Crusca, 1582) et ailleurs. II eut deux conséquences. D'une part, les frontières nationales sont devenues des frontières linguistiques par la force de structuration de l'enseignement, d'autre part l'étatisation de la langue déployée comme un symbole national et historique eut pour effet de la rigidifier.

On ne peut qu'imparfaitement décalquer ce processus sur la construction européenne dans la mesure où la notion de frontière qui disparaît entre les États membres pour se fixer à la périphérie de l'Union est plus liée à l'idée du contrôle des échanges humains et marchands avec le reste du monde qu'à l'approfondissement de «l'Européanité», que l'on pourrait concevoir comme une version du nationalisme rénovée par la cosmopolitique (cf. Habermas 2000). La notion d'espace d'éducation européen est encore trop embryonnaire pour que l'on imagine le principe de domination d'une langue unique car celle-ci serait encore assimilée à une langue nationale.

On retrouve en revanche le besoin de réduire, comme au stade de la formation des États nations puis de leur modernisation, les problèmes d'intelligibilité mutuelle entre les langues parlées sur un territoire unifié politiquement. À l'époque, les problèmes étaient posés par le caractère limité des échanges entre les personnes du fait de la non généralisation de l'école et du faible déplacement des populations qui voyageaient moins qu'aujourd'hui (hasard, coût, pénibilité et insécurité des transports). Aujourd'hui, la libre circulation des personnes sur le territoire de l'Union et le réseau des transports facilitent les déplacements de la fraction de la population qui en a l'usage, ce qui concerne plutôt les élites, une partie de 
la communauté étudiante et universitaire et les touristes. Peu d'Européens encore ont une connaissance directe des États dont ils ne sont pas citoyens.

L'accès différentiel et inégal au savoir et aux transports favorise l'émergence d'une langue parlée au centre, occupé par les élites qui se détachent des langues parlées dans les régions. Ce centre peut désigner le centre du pays pour ce qui concerne la fabrication de l'état, le centre des cultures (si l'on se souvient du rôle du français au $18^{\mathrm{e}}$ siècle comme langue de culture au niveau des élites européennes et dans les cours des monarques) ou le centre des affaires, ce qui serait plus conforme à l'époque actuelle. En ce qui concerne le fonctionnement de l'Europe, l'identification d'un centre unique est discutable, car pour important que soit Bruxelles comme lieu de lobbying, le centre européen doit négocier avec chacun des centres nationaux dans la langue de ces Etats (Règlement no $1 \mathrm{du}$ Conseil des Ministres). Pourtant, un jargon européen s'invente dans les institutions intégrées, comme à la Commission, qui se diffuse dans les administrations nationales et dans les espaces de communication animés ou arbitrés par les institutions européennes. On pourrait donc y voir l'embryon de formation d'une langue de communication européenne.

Les développements historiques aboutissant à la formation du français, de l'allemand, de l'espagnol, etc. sont liés au fait que les élites centrales gagnaient à voir les élites régionales faire l'effort d'apprendre la langue centrale, parlée à la capitale, et assurer la médiation nécessaire. La situation a commencé de changer lorsque les élites régionales ont perdu la clientèle des gens pour qui elles assuraient la médiation linguistique, correspondant au moment où, grâce à la diffusion de l'école, on a commencé de parler la langue centrale, ce qui a marqué le début du déclin des langues régionales. Cela put résulter de deux tendances, soit que les élites locales aient perdu la bataille politique - ce qui fut le cas en France avec le triomphe de la centralisation républicaine et du modèle jacobin -, soit qu'elles se soient rangées pour propager la langue dominante, mettre en place des écoles et participer au processus de construction nationale. La langue centrale comme moyen de coordination sociale dans la fabrication de la société nationale pourrait jouer le même rôle dans l'espace européen. On pourrait ainsi voir l'anglais devenir une langue centrale au niveau européen, par le double fait qu'elle serait le médium de communication principal - usité en tout cas dans le monde des affaires et comme seconde langue commune - et aussi la langue maîtrisée par les individus qui n'ont plus besoin de s'appuyer sur leurs 
élites nationales pour dialoguer avec le centre, en l'occurrence Bruxelles. Cela résulterait d'une part des choix retenus par les États dans leurs systèmes d'éducation et d'autre part des différents mécanismes par lesquels la gouvernance européenne modifie les formes nationales de faire le politique.

Comme on constate qu'historiquement la centralité d'une langue a abouti à un processus d'officialisation propice à la cristallisation des identités nationales, (le principe "Une langue un État» se substituant au principe consacré par la Paix d'Augsbourg "cujus regio ejus religio») la question se pose de savoir si la centralité de l'anglais en Europe s'accompagnerait d'un processus d'identification de type supra-national. Pour l'instant on constate une très grande faiblesse du sentiment d'identification à l'Europe, ce qui invite à réfléchir sur la désolidarisation du rapport entre langue et identité. On peut donc imaginer avoir en Europe une langue de communication qui ne serait pas une langue identitaire.

En effet, durant l'évolution vers la reconnaissance d'une langue nationale comme en Allemagne, Espagne, Italie, France, etc., les États sont devenus les grands protecteurs des langues officielles pour des besoins précis : l'écriture de la loi qui suppose l'acceptation de formes de standardisation et la stabilisation du sens des mots; la transaction avec les administrateurs chargés de mettre en œuvre et de faire respecter la loi; l'instruction scolaire; le développement des affaires entre le centre et les locaux. Mais la langue devenue centrale pour des raisons de management étatique a tendance à déborder du sillon ainsi tracé. Associée au prestige et à l'aisance, elle est aussi convoitée par les individus qui y voient un moyen d'améliorer leur sort. Cela se répercute via les mass media contrôlés par les autorités, ce qui affecte la langue de la population. Ces facteurs sont transposables au niveau européen pratiquement dans les mêmes termes sous trois réserves majeures. D'une part, l'instruction scolaire s'inscrit aujourd'hui dans une dynamique d'ouverture à la diversité européenne plutôt que de fermeture nationale. D'autre part, le développement des affaires ne vise pas simplement les échanges entre les locaux via un centre habilitateur (Bruxelles) mais également avec le reste du monde déjà lié par l'histoire à chacun des centres nationaux, ce qui pose le problème de la liaison à construire entre la politique commerciale européenne et la politique étrangère et de sécurité commune. Enfin, la construction européenne ne mobilise pas d'enjeu de type nationaliste, ni sentiment patriotique, ni ferveur, ni volonté de mourir pour la sauver. On doit donc présenter une autre vue des évolutions linguistiques en Europe en approchant la question par l'analyse des systèmes de communication et des pratiques relatives à la gouvernance. 


\section{Langue et gouvernance}

La rigidité de l'identification par la langue nationale est attaquée par le besoin de se projeter dans un espace qui n'est plus confiné au territoire national mais s'étend à l'ensemble de l'Europe. L'Union européenne procède sans doute d'une manière qui rappelle le processus antérieur d'unification des régions dans le cadre national, mais elle ne présente pas moins des différences majeures au regard des pratiques de gouvernance. Ce qui limite fortement sa possibilité d'officialiser une seule langue.

Tout d'abord, le processus se déroule à l'échelon supérieur des États dont la capacité de mise en œuvre administrative des directives européennes, dans leurs langues et selon leurs procédures, n'est pas contestée. Ensuite, c'est essentiellement le développement des affaires lié à la mise en œuvre du marché unique, de l'espace économique européen qui induit des besoins d'harmonisation, y compris linguistique, dans les domaines allant du monde des entreprises au marché du travail, en passant par l'éducation, le commerce et la finance. Enfin, l'unification «par le haut" s'accompagne d'un processus de reconnaissance de la diversité "par le bas". La situation actuelle de l'UE présente donc des caractéristiques originales. L'intégration européenne fragilise le rapport entre l'État et la nation par la voie des transferts de souveraineté qui déplacent le centre de la décision des capitales nationales vers Bruxelles. Simultanément, la voie des décentralisations et des délégations de compétences qui accompagnent la construction d'une Europe des Régions est propice à la revitalisation des sentiments infra-nationaux et des parlers régionaux. En conséquence, bien que d'un côté, la plupart des langues modernes occidentales se soient consolidées par l'acquisition d'un statut hégémonique dans un pays, ce qui explique le terrain des rivalités entre les États européens aujourd'hui, de l'autre, la situation de marginalisation des langues régionales n'est plus aussi incontestable qu'autrefois. II en résulte que le paysage des langues officielles et nationales bouge à mesure que s'affirment les droits linguistiques des minorités nationales, régionales et ethniques. Ainsi, le cadre unitaire de l'Europe, hérité du cadre idéologique de l'État nation, devrait-il être repensé dans la perspective d'un fonctionnement de type pluraliste, à l'indienne.

Le passage du statut d'État nation, indépendant et souverain, à celui d'État membre d'une Union dans laquelle s'organisent des transferts de souveraineté, suppose que soient assumés collectivement, par les gouvernements qui les négocient et par les représentants des peuples qui les ratifient, les changements politiques et économiques et, aussi, le repli des références culturelles et symboliques jusqu'alors utiles à la fabrication du 
sentiment national. La suppression des frontières intérieures qui accompagna la mise en place du marché unique en 1992 et le passage à l'Euro au premier janvier 2002 représentent, pour les citoyens, deux étapes les plus visibles de la mise en place de l'Europe et de dépassement du cadre national. Mais l'adoption d'une langue commune - en l'occurrence, l'anglais - est, elle, perçue comme une entreprise illégitime, portée par des Eurocrates déconnectés des réalités du terrain, servant tout au plus les intérêts des Britanniques dont le sentiment européen est, paradoxalement, très bas au niveau populaire. Ce refus est lié à l'argument précédemment exposé selon lequel la langue reste représentative de l'État dans lequel elle a été officialisée. Or l'Union est un club d'égaux, non une série d'États dominés par un plus puissant. Pour sortir de ce dilemme, il faut déplacer le débat linguistique européen du niveau des rapports entre centre et périphérie (question de l'officialité) vers la relation entre monolinguisme et multilinguisme (question de pratique).

Pour Sue Wright (1999), sociolinguiste britannique, le fait que l'on ne parle pas de langue commune dans l'Union européenne est cohérent avec le fait qu'il est commun de ne pas parler de la différence des langues, la grande absente de l'histoire des États et de leurs relations. On sait en effet peu de chose sur la manière dont la différence de langues peut avoir affecté la conception, la rédaction et la signature des grands traités, ceux de Versailles, de Trianon ou de Sèvres par exemple. On ne connaît pas le détail des situations d'interlocutions qui forgent les grandes ententes ou les inimitiés. L'UE manque d'un débat sur la gestion de la communication dans une Europe plus intégrée et pratiquement aucune réflexion n'accompagne la mise en place d'un espace politique qui dans l'état actuel des connaissances des 375 millions d'Européens devrait être multilingue. Tout cela se réalise sur une base pragmatique, à charge pour les individus de résoudre leur problème linguistique.

Wright s'intéresse à la transformation des espaces de communication qui, en devenant plus fluides, rendent le besoin d'une langue unique plus pressant. Langue qui peut être le vecteur d'une identité choisie, pas nécessairement nationaliste, ce qui lui permet d'affirmer qu'il faut admettre le fait de la dominance de l'anglais et cesser de croire que l'UE est multilingue. Elle fait donc la théorie d'une régression historique du multilinguisme vers le monolinguisme à l'échelle européenne. Un monolinguisme qui serait le legs des Etats à l'Union, comme une fatalité qu'il lui faudrait assumer.

À ses yeux, l'absence de débat relève d'un manque de volonté politique car la langue n'est pas seulement un moyen de communication mais 
le signe d'une appartenance à la communauté9. Deux siècles de domination du principe «Un peuple, une langue, un État»10 sont responsables de ce que la plupart des Européens acceptent le principe d'une seule langue nationale, ce qui fait qu'il est impossible de concevoir qu'une langue nationale soit éclipsée sur la scène européenne où se jouent des débats publics. Mais il également impossible d'imaginer que tous les citoyens résolvent par leurs propres moyens la question du bilinguisme inhérent au fait que l'Europe est une communauté d'États membres, parlant chacun des langues différentes. Il en résulte que le polyglottisme actuellement en Europe est le fait soit des élites, soit des membres de minorités qui refusent l'assimilation dans la langue nationale qu'elles ont été obligées de maîtriser.

Comme nous l'avons remarqué plus haut, les facteurs qui ont eu pour effet de consolider la langue nationale peuvent être observés aujourd'hui dans le processus de la construction européenne. On retrouve la fonction des frontières pour le contrôle du commerce et la captation des taxes et douanes, déplacées du territoire national à la périphérie de l'union. Le besoin de l'administration de communiquer sur le territoire de sa compétence dans la langue comprise par les sujets est au cœur des questions relatives à la mise en œuvre des directives européennes. Dans l'Union comme autrefois dans le cadre national, s'organise l'intégration politique des espaces à travers le développement d'élections supranationales et la construction d'un espace de citoyenneté. On voit enfin lentement émerger des médias européens ayant pour objet de rendre l'Europe plus visible. Le processus qui a renforcé les langues nationales au détriment des langues régionales travaille, dans le contexte de la construction européenne, à la marginalisation des langues nationales au profit d'un nouveau monolinguisme qui s'insinue là où devraient se développer des échanges multilingues.

9. Comme on l'a vu plus haut, les termes par lesquels on réfléchit à l'Union européenne empruntent au modèle qui a précédé l'invention de cet objet politique. On dispose d'une illustration toute récente de ce mécanisme avec les réactions de la France à la proposition de constitution européenne que G. Shroeder présentait devant son parti le 12 mai, qualifiée de vision très allemande.

10. Le processus d'unification linguistique en France part de la révolution. Au début, les tracts et les documents étaient traduits, puis les révolutionnaires révisèrent la position pour déclarer que le français sera le langage de la révolution et de la nation «La monarchie avait des raisons de ressembler à la tour de Babel, dans la démocratie laisser les citoyens ignorants de la langue nationale, incapables de contrôler le pouvoir, c'est trahir la patrie, c'est méconnaître les bienfaits de l'imprimerie" (Barère in Lodge) (Wright 1999). Ensuite la conscription jouera un grand rôle pour éduquer les jeunes hommes provinciaux et l'école, en devenant obligatoire, étendra l'éducation en français. C'est le moment de la répression physique de la langue régionale et de l'identité locale et de la domination du modèle républicain de l'unité de la langue et de la république. 
Avec l'Union européenne, deux lingua franca se sont développées; le français parce que les institutions sont situées en territoire français et en raison du prestige de la langue, l'anglais, en raison de son rôle global dans le monde. Mais l'anglais est devenue la première langue de travail et de communication que l'on utilise dans les programmes de l'UE ou dans les institutions que met en place l'Union pour développer les échanges en réseau. Elle sert de langue relais dans toutes les situations de grand écart linguistique. Elle occupe une position si centrale que Romano Prodi, actuel président de la Commission européenne, voudrait l'adopter comme unique langue de travail, tandis que les conservateurs britanniques souhaiteraient en faire l'unique langue pivot du système de traduction / interprétation au Parlement européen. De fait, on note sur le territoire des Quinze Etats membres une progression sans égal de l'anglais : plus de $80 \%$ des Européens apprennent l'anglais dans le secondaire, et en Espagne par exemple, on assiste à la substitution quasi complète de l'enseignement du français par l'anglais.

En même temps, à l'arrière plan des rivalités entre grandes langues dominantes et des tendances à recréer un monolinguisme officiel, on assiste à la renaissance des langues régionales, ce qui a pour effet de démontrer le caractère transitoire des politiques nationales d'unification. En plusieurs points de l'Union, des forces centrifuges sont à l'œuvre comme on le voit avec l'adoption de la constitution belge qui reconnaît un Etat fédéral fait de régions et de communautés, la question des autonomies en Espagne, le statut de la Corse en France, etc. L'affaiblissement de l'État nation comme base du pouvoir dans le contexte de l'unification européenne a pour conséquence un double phénomène qui prend les langues nationales en ciseau par l'émergence d'une lingua franca au sommet - en l'occurrence l'anglais - et par la renaissance des formes locales et des langues régionales. L'ensemble européen présente donc une situation type de multilinguisme qui s'appuie sur une multiplicité de situations locales de diglossie, un terme qui désigne dans un contexte de bilinguisme l'inégalité de statut entre deux langues.

Ces phénomènes de diglossie, de multilinguisme obligatoire ou accepté, de fluidité linguistique changent la perspective de l'expression des citoyens européens par la seule langue nationale et le principe de leur identification politique à un seul drapeau. Être européen n'a pas de correspondance en termes de parler européen si ce n'est sous forme d'un multilinguisme plus ou moins bien maîtrisé dans lequel les langues régionales rivalisent avec les grandes langues nationales. Le monolinguisme 
n'est donc pas une fatalité que l'Union doit assumer. Le partage du pouvoir de l'État avec des autorités locales et des autorités supra-nationales remet en cause le concept de souveraineté linguistique, ce qui met en tension chacune des langues officielles. Ces évolutions doivent être mises au crédit de l'approfondissement de la démocratie qui accompagne le mouvement d'unification européenne par ailleurs fort décriée comme étant «la chose des bureaucrates».

Le retour des langues minoritaires sur la scène officielle.

En 1982, Bruxelles - ville qui symbolise le centre de l'Europe - voyait la naissance d'un Bureau Européen des Langues les Moins Répandues (BELMR), grâce à la résolution Arfé du Parlement européen. L'entreprise se développait en 1983 avec une demande faite à la Commission de poursuivre ses actions en faveur de ces langues, le vote d'un budget et la naissance d'un intergroupe. La représentation des peuples européens qui venait tout juste de se voir reconnaître le droit d'être élue au suffrage direct (1979) se faisait l'écho de demandes qui, jusqu'alors, étaient bloquées par les États au niveau officiel. En 1984, la conférence permanente des pouvoirs locaux et régionaux (CPPLR) du Conseil de l'Europe organisait une séance publique sur ces questions, et produisait l'esquisse d'une idée de charte dont le projet devait être préparé par le BELMR. En 1987, la résolution de Kuijpers proposait aux États membres et à la Commission des actions concrètes. En mars 1988, la CPPLR adoptait le texte de la charte européenne et en octobre l'assemblée parlementaire proposait de lui donner le statut d'une convention européenne, ce qui sera voté par le Comité des Ministres du Conseil de l'Europe en juin 1992. Le 5 novembre 1992, était ouverte une convention pour la signature de la charte qui était signée par quinze pays : Allemagne, Autriche, Danemark, Espagne, Finlande, Luxembourg, Pays Bas, Chypre, Hongrie, Liechtenstein, Malte, Norvège, Suisse, Roumanie et Ukraine. Mais très peu de pays devaient la ratifier pour qu'elle s'applique dans la législation interne. En 2000, s'étaient impliqués seulement 9 pays sur quarante dont Finlande, Norvège, Pays Bas et Hongrie et aucun des grands pays concernés par la répression des langues régionales.

En 1994, le Parlement Européen votait un soutien à la Charte Européenne des Langues Régionales ou Minoritaires, invitait les États à la ratifier, demandait la mise en place par la Commission, pour les langues minoritaires, d'un programme inspiré par LINGUA ainsi que la publication de la carte scientifique des langues les moins répandues. II demandait aussi que le Conseil et la Commission encouragent les orga- 
nismes représentant les langues minoritaires ${ }^{11}$.

Ces entreprises, favorables à la diversité linguistique de l'Union, suscitèrent des réactions mitigées de la part de la France qui se méfie des risques de discrimination positive en faveur de ces langues dans les territoires où elles sont parlées, la charte impliquant la possibilité qu'elles soient utilisées par l'administration, les services publics et la justice, tandis qu'elle impose à l'État d'organiser l'enseignement de ces langues ${ }^{12}$. La France résiste au fait que les mêmes droits soient conférés aux langues régionales et aux langues minoritaires qui sont certes parlées par un nombre significatif de Français mais qui ne sont pas langues officielles dans un pays. Cela désigne les langues que les citoyens d'origine étrangère utilisent dans leur vie privée, ce qui est, par exemple, le cas du kabyle par rapport au français et à l'arabe. Mais elle concède sans difficulté aujourd'hui l'usage dans la vie privée, l'enseignement public et privé des langues régionales ainsi que le droit d'écrire ses journaux et de présenter des émissions radiophoniques et télévisuelles (qu'elles soient transmises par les réseaux hertziens, le câble ou le satellite) sur le service public et privé. La ratification de la charte fut remise en question par certains représentants politiques, au motif qu'elle serait contraire à l'article 2 de la constitution qui stipule que «la langue de la république est le français». Mais la constitutionnalité du texte a été validée et la charte ratifiée en 1999. Bien que l'État considère toujours que l'avenir des jeunes est mieux assuré par la promotion du français et l'apprentissage des langues étrangères que par le repli sur des idiomes respectables mais pratiqués dans un espace restreint, le ministère de l'éducation nationale fait quelques efforts pour mettre en œuvre ses engagements internationaux.

11. Le 10 novembre 1994, le comité des ministres du Conseil de l'Europe adoptait la convention cadre pour la protection des minorités nationales et validait le principe de la sauvegarde et le développement des droits de l'Homme et des libertés fondamentales. Celles ci comprennent la liberté linguistique et le droit d'apprendre une langue minoritaire et de recevoir un enseignement dans cette langue. Le document a été signé par Albanie, Allemagne, Autriche, Chypre, Danemark, Espagne, Estonie, Finlande, Hongrie, Irlande, Islande, Lettonie, Liechtenstein, Lituanie, Luxembourg, Malte, Moldavie, Norvège, Pays-Bas, Pologne, Portugal, République Slovaquie, République Tchèque, Roumanie, Royaume-Uni, Saint Marin, Slovénie, Suède, Suisse et Ukraine. Mais seulement quatre pays ont ratifié la convention cadre : Hongrie, Roumanie, Slovaquie, Espagne

12. Depuis l'Edit de Villers Coterêts, le français est utilisé dans le domaine de la Justice, mais l'utilisation sans interprète est tolérée pour le basque, le corse, l'allemand et l'alsacien, si le juge connaît la langue des parties. La langue régionale peut être utilisée dans les conseils municipaux si le compte rendu est rédigé en français. Pour l'administration générale, le français est de règle mais les fonctionnaires peuvent utiliser oralement leur langue, et la poste accepte les adresses écrites dans la langue régionale. 
De facto, et pour ne retenir que la situation française, la construction d'une langue standard à vocation officielle a abouti à ce que l'on ait une très grande méconnaissance des langues parlées sur le territoire français. Selon le directeur de l'Institut National de la Langue Française, soixante quinze langues sont considérées comme langues régionales ou historiques, dont cinquante et une sont parlées dans les Départements et les Territoires d'Outre Mer (DOM TOM) et vingt quatre dans l'Hexagone ${ }^{13}$. Dans les TOM DOM, à l'exception du tahitien, aucune langue ne bénéficie d'un enseignement dans les écoles, un droit qui, en métropole, a été validé par différentes lois depuis $1951^{14}$.

Aujourd'hui l'enseignement des langues régionales (LR) est proposé sur la base du volontariat, avec une généralisation progressive de l'enseignement. En 2000-2001, sur dix millions d'élèves, 152500 ont reçu un enseignement partiel en LR, 28500 écoliers et collégiens ont reçu un enseignement bilingue. Mais les spécialistes ne constatent pas de bilinguisme scolaire. De fait, des langues identitaires, comme l'hébreu, ou lointaines, comme le chinois, ont plus de succès auprès des élèves et des parents du secondaire que les langues maternelles des populations concernées. L'accès est aussi très inégal selon les communautés, les enseignements en LR les plus répandus se faisant en occitan, breton, corse et catalan, le problème étant d'adapter la formation des enseignants dans ces langues (basque, corse, breton, catalan, créole, langue d'oc, ou langues régionales d'Alsace et Moselle) en même temps que d'améliorer la connaissance des langues communautaires. Face au recul des langues régionales et au problème que posent les langues d'immigration dans l'État unitaire français, on note un frémissement du côté ministériel. Le 26 avril 2001, Jack Lang faisait état de nouvelles résolutions pour développer

13. Ces langues sont l'alsacien (900 000 locuteurs), le francique lorrain (350 à 500000 ), le flamand (20 à 40000 locuteurs), le breton (200 à 300 000), l'occitan (3 millions de personnes capables de soutenir une petite conversation) qui porte différents noms selon les régions, (provençal, auvergnat, limousin, languedocien, gascon, béarnais,). II faut ajouter le basque (40 à 100000 locuteurs), le catalan (126 000), le corse (50\% de la population, soit moins de 150000 locuteurs), le franco provençal - savoyard (quelques milliers de locuteurs et les langues d'oïl (picard, gallo, poitevin, saintongeais, normand, morvandiau, champenois) en voie de disparition.

14. La loi Deixonne 1951 (abrogée) scelle la reconnaissance du droit à l'existence des langues régionales et la possibilité d'un enseignement facultatif et limité pour le breton, le basque, le catalan et l'occitan . La loi Haby (1975) introduit le droit à l'enseignement des langues et cultures régionales tout au long de la scolarité. La loi Bas-Auriol (1975, abrogée par Toubon en 1994) rétablit une protection de la langue française par la volonté de la protéger contre les abus de l'unilinguisme (anglais) sur les produits de consommation. La loi Toubon de 1994 en vigueur, consacre cette défense du français face à l'anglais et ne s'oppose pas aux dispositions en vigueur pour les langues régionales. 
l'enseignement des LR afin de "réparer une injustice historique qui consistait à les éradiquer", "accomplir un acte de reconnaissance de la dignité de ces cultures" et parier sur une nouvelle dynamique. En ce qui concerne les langues des immigrants, des accords ont été signés avec le Portugal, l'Italie, l'Espagne, le Maroc, la Yougoslavie, la Turquie, l'Algérie pour prévoir des cours dans les heures scolaires. Simultanément, la France s'efforce d'assumer ses engagements pour développer l'enseignement des langues communautaires (européennes) et la connaissance de plusieurs langues de communication internationale s'impose d'ores et déjà à l'entrée dans certaines grandes écoles.

La scène linguistique française pourrait donc être caractérisée de multilingue, ce qui semble tout à fait paradoxal pour un État qui, aux yeux du monde, semble si attaché au principe de l'unité de la langue. On ne peut, en effet, négliger les occurrences significatives de communication plurilinguistique, dans lesquelles langues régionales et langues européennes, c'est-à-dire les langues officielles des États voisins, se joignent au français pour informer les individus se déplaçant ou vivant en France. Un système plurilingue n'est pas également répandu sur le territoire de la république, mais il se développe progressivement, à partir de réactions manifestant soit l'incompréhension devant la seule information en français (cas type du touriste égaré dans une gare), soit le refus de celle-ci pour des raisons politiques (signalétique routière dans les régions à velléité autonomiste). Selon des combinaisons linguistiques différentes coexistant avec le français, il est réalisé avec deux langues européennes dans les grandes métropoles, notamment dans les nœuds de communication, (gares ferroviaires, routières, maritimes, ports et aéroports) et dans la langue régionale des pays (communes, département, régions). La liberté régnant dans le domaine de l'audiovisuel et de la presse (et le succès de la presse régionale) a aussi considérablement transformé le rapport entre langue nationale et langues minoritaires et régionales.

\section{Conclusion}

On voit donc que la situation linguistique sur le terrain évolue considérablement depuis une quinzaine d'années, en France comme dans les autres États membres de l'Union. II nous faut donc revenir au problème que représente la diversité linguistique dans la fabrication d'une politique commune.

La multiplicité actuelle des langues officielles et l'accroissement futur, correspondant à l'intégration des nouveaux États membres, fait craindre une paralysie du fonctionnement des institutions incapables de gérer cet 
impact linguistique moins en termes budgétaires qu'en termes organisationnels. Le débat linguistique qui peine à se mettre en place, tant les représentants politiques nationaux refusent d'assumer les choix d'une simplification impopulaire, si ce n'est dans leur propre électorat si la langue nationale était retenue, du moins chez les voisins dont la langue ne serait pas retenue, reflète la difficulté à convertir un modèle unitaire en fonctionnement pluraliste.

Le modèle unitaire, hérité de la formation et de la modernisation de l'État nation européen, aboutit d'une part à la reconnaissance des langues officielles et donc à leur rivalité aujourd'hui sur le terrain européen, d'autre part au développement du monolinguisme au détriment du multilinguisme. Ce choix n'est pas nécessairement une fatalité que l'Union européenne devrait assumer. La reconnaissance du fait pluraliste européen, à savoir que l'Union est essentiellement composée d'États membres, souverains et égaux qui, ensemble, décident d'approfondir leur intégration, peut s'accompagner d'une décision politique visant à donner aux ressortissants européens, citoyens nationaux et européens, les moyens de s'exprimer sur plusieurs registres. Cela leur permettrait, entre autre, de mieux comprendre les enjeux d'une Europe qui ne serait pas simplement économique, comme elle l'a été jusqu'à présent. L'apprentissage de plusieurs langues communautaires, recommandé par la Commission en 1995, et mis en œuvre dans la plupart des États est le signe d'une évolution vers un plurilinguisme généralisé. Mais les Européens sont encore loin de maîtriser les trois langues communautaires susceptibles de faciliter leur déplacement sur le territoire de l'Union et leur insertion sur différents marchés du travail.

Parallèlement, les États, sous l'égide du Conseil de l'Europe, progressent vers la reconnaissance des langues minoritaires et régionales, ce qui accrédite la thèse du développement du multilinguisme européen. Certains voient ces évolutions comme un retour vers le passé qui irait de pair avec le développement de nouvelles féodalités fondées sur le développement de réseaux personnels (Bull, in Wright). Mais on pourrait tout autant concevoir ce mouvement comme un fruit de la démocratie visant à généraliser une faculté jusqu'alors réservée aux élites.

Les responsables politiques ont du mal à développer la communication dans un cadre défini par la pluralité des idiomes qu'il s'agisse des langues officielles, des langues parlées, des langues régionales et de toutes les variantes enregistrées dans les États européens. Ils peinent à faire les choix et à dépassionner le rapport entre langue et identité. Mais 
l'identification par la langue n'est-elle pas due à un ressort ancien, bien connu de la sociologie et inhérent à la construction des nationalismes qui veut que l'on s'identifie par sa manière de parler. La maîtrise de plusieurs langues dans un espace de communication qui, dans le cadre européen, s'élargit aux voisins, serait, mieux que l'imposition d'une langue unique, même centrale dans le fonctionnement des institutions et des individus, susceptible d'approfondir la construction d'un espace de concitoyenneté. Être européen pourrait alors correspondre à la faculté jusqu'à présent maîtrisée par les Euro-fonctionnaires, la jet set internationale, et quelques autres, de parler plusieurs langues sans que leur identité nationale ait à souffrir de leur compétence.

Ces évolutions ne ressortissant pas seulement du politique, la technologie pourrait permettre de penser des systèmes de communication qui visent à faire progresser la société de la connaissance qu'invoque la Commission européenne. Les fameuses «nouvelles technologies de l'information et de la communication" dont les développements signent le début de l'ère post-industrielle ne pourraient-elles enrayer l'érosion de la diversité et faciliter l'insertion des individus dans un espace de communication multilingue, susceptible de s'étendre mais dont ils ne maîtrisent pas tous les codes (De Cilla; Krumm et Wodak 2001)? La réponse viendra de ceux qui ont intérêt à ce qu'une langue dominante ne règne pas seule sur le terrain de la communication européenne, à condition qu'un débat soit ouvert largement et qu'ils puissent faire entendre leurs voix.

\author{
Irène Bellier \\ Laboratoire d'Anthropologie \\ et des Institutions et des Organisations Sociales \\ LAIOS-CNRS \\ Paris
}




\section{Bibliographie}

Anderson Benedict (1983) Imagined Community, London : Verso.

Abélès Marc (1992) La vie quotidienne au Parlement Européen, Hachette, Paris.

Abélès et Bellier, (1996) «La Commission Européenne : du compromis culturel à la culture politique du compromis", Revue Française de Science Politique, vol 46, no 3, 431-455.

Appadurai Arjun (1996) Modernity at large, Cultural Dimensions of Globalization, New Deli, Sage University Press, et Oxford, Oxford University Press.

Bellier Irène (1999 a) «European Institutions and Linguistic Diversity : a Problematic Unity" in National Identities and Regional Cooperation : experiences of European Integration and South Asia Perceptions, Chopra H.S, Frank, R. Schröder, J., with Dorin B. \& Kueck, Manohar, New Delhi. (1999 b) «De la fonction de chef à celle de médiateur», Les Cahiers de l'ENSPTT, Les Réseaux de dirigeants, $n^{\circ} 11,35-41$.

(2001) «Between 'Transparency' and 'opacity' : Official Communication in New Europe", in Rudolph de Cillia, Hans-Jürgen Krumm, Ruth Wodak (Hrsg) Loss of Communication in the Information Age, Wien, Autriche.

Born Joachim "O Futuro das Linguas Estransgeiras em Instituçoes Internacionais : Uniao Européiae Mercosul», Revista da Comunicaçao, $\mathrm{N}^{\circ}$ 21 96/2 : 41-52.

Cillia Rudolph de, Krumm Hans-Jürgen, Wodak Ruth (Hrsg) Loss of Communication in the Information Age, Academy des Sciences Sociales, Wien, Autriche.

Commission Européenne (1995) Livre Blanc sur l'Éducation et la Formation «Enseigner et Apprendre, Vers la Société Cognitive» (http://europa.eu.int)

Habermas Jurgen (2000), Après l'État nation. Une nouvelle constellation politique, Fayard, Paris.

La Guérivière Jean de (1992), "Voyage à l'intérieur de l'Eurocratie», Le Monde Éditions, Paris.

Mullen Paul Fabien (2000), "So you hear what I hear? Translation, Expansion, and crisis in the European, Court of Justice", in Maria Green Cowles and Michael Smith, The State of the European Union, vol 5, Risks, Reform, Resistance and Revival, Oxford, OUP. 
Swaan, Abram de (1995) The language constellation of the European Union. A perspective from the political sociology of language. Inclus dans un livre à paraître en octobre 2001 "The world language system», Polity Press.

Wright Sue (1999) «A Community that can communicate? The linguistic factor in European integration", in Dennis Smith and Sue Wright, Whose Europe? The turn towards democracy, Blackwell Publishers, Oxford. 